\title{
RESEARCH
}

Open Access

\section{Blast traumatic brain injury and serum inflammatory cytokines: a repeated measures case-control study among U.S. military service members}

Jennifer Rusiecki ${ }^{1 *}$, Lynn I. Levin², Li Wang ${ }^{1}$, Celia Byrne ${ }^{1}$, Jayasree Krishnamurthy ${ }^{3}$, Ligong Chen', Zygmunt Galdzicki ${ }^{4}$ and Louis M. French ${ }^{5}$

\begin{abstract}
Background: There is a paucity of human data on exposure to blast traumatic brain injury (bTBI) and the corresponding systemic cytokine immune response at later time points (i.e., months, years) post-injury.

Methods: We conducted a repeated measures, case-control study, examining associations of serum levels of proand anti-inflammatory cytokines, measured both pre- and post-deployment with having mild and moderate/severe bTBI. Utilizing serum from the Department of Defense Serum Repository cytokines were measured via an ELISAbased array for 15 cytokines. We compared pre- vs. post-levels among mild cases, moderate/severe cases, and controls and carried out case-control comparisons, using paired t- tests and generalized linear models.

Results: The average time between bTBI and post-deployment/bTBI serum among cases was 315.8 days. From preto post-deployment/bTBl, levels of interleukin 8 (IL-8) were decreased among both mild cases $(\mu=-83.43 \mathrm{pg} / \mathrm{ml}$; s.e. $=21.66)$ and moderate $/$ severe cases $(\mu=-107.67 \mathrm{pg} / \mathrm{ml}$; s.e. $=28.74 \mathrm{pg} / \mathrm{ml})$, while levels increased among controls $(\mu=32.86 \mathrm{pg} / \mathrm{ml}$; s.e. $=30.29)$. The same pattern occurred for matrix metallopeptidase 3 (MMP3), with levels decreasing for moderate/severe cases $(\mu=-3369.24 \mathrm{pg} / \mathrm{ml}$; s.e. $=1701.68)$ and increasing for controls $(\mu=1859.60$ $\mathrm{pg} / \mathrm{ml}$; s.e. $=1737.51)$ from pre- to post-deployment/bTBI. Evidence was also suggestive of case-control differences, from pre- to post-deployment/bTBI for interleukin 1 alpha (IL-1a), interleukin 4 (IL-4), and interleukin 6 (IL-6) among moderate/severe cases.
\end{abstract}

Conclusion: The findings of this longitudinal study indicate that in the chronic phase of bTBI, levels of IL-8 and MMP3 may be substantially lower than pre-injury. These results need confirmation in other studies, potentially those that account for treatment differences, which was not possible in our study.

Keywords: Traumatic brain injury, TBI, Cytokines, Inflammation, Operation Iraqi freedom, Operation enduring freedom, Protein, Microarray, Military

\footnotetext{
*Correspondence: jennifer.rusiecki@usuhs.edu

'Department of Preventive Medicine and Biostatistics, F. Edward Hébert

School of Medicine, Uniformed Services University, Bethesda, MD, USA

Full list of author information is available at the end of the article
}

(c) The Author(s). 2020 Open Access This article is distributed under the terms of the Creative Commons Attribution 4.0 International License (http://creativecommons.org/licenses/by/4.0/), which permits unrestricted use, distribution, and reproduction in any medium, provided you give appropriate credit to the original author(s) and the source, provide a link to the Creative Commons license, and indicate if changes were made. The Creative Commons Public Domain Dedication waiver (http://creativecommons.org/publicdomain/zero/1.0/) applies to the data made available in this article, unless otherwise stated. 


\section{Background}

During more than 10 years of the global war on terror (2000-2011), approximately 290,000 US military personnel suffered traumatic brain injury (TBI); nearly $70 \%$ of these were due to explosive blast exposure [1, 2]. Blast-associated TBI (bTBI) comprises four injury methods: primary (blast overpressure waves), secondary (trauma from shrapnel/objects), tertiary (head trauma with blunt impact from blast wind), and quaternary (thermal burns, toxic chemicals) [3-5]. Primary non-penetrating blast injuries result from the direct effects of complex pressure waves generated by an explosion, known as blast overpressure (BOP) injuries [6]. Primary blast is exceedingly rare, and bTBI usually has secondary and/or tertiary components to it $[7,8]$. In contrast, non-penetrating, nonblast TBI are closed head injuries such as sports-related concussions or blunt force trauma [9]. Although explosive, non-penetrating bTBI shares many clinical features with non-blast TBI [10] and the symptoms often appear the same clinically; there are other features, including neuroimaging and histopathology, as well as animal study results that suggest important differences [10-14]. Taken together, these findings support the view that bTBI may be a separate (unique) form of TBI, with distinct characteristics and etiology.

Inflammation plays a central role in the pathogenesis of secondary injury after TBI $[15,16]$. Injury activates cells in the central nervous system such as glial cells, microglia, and astrocytes and causes secretion of inflammatory cytokines, chemotactic cytokines, and glycoproteins (including the metallopeptidase (MMP) family of proteins). This drives the increased deposit of parenchymal and peripheral immune cells in the target brain region with the compromised blood brain barrier (BBB) $[17,18]$ Cells in the periphery also produce cytokines and chemokines that are involved in both local and systemic immune responses $[16,18-21]$. The release of pro- and anti-inflammatory cytokines varies, based on the timing after injury [22, 23], with molecular changes activating further downstream systemic immune responses, resulting in both reactive and restorative inflammation processes [16, 21, 24].

Clinical research on serum cytokine levels and TBI severity (mild, moderate, and severe) has mainly centered on the acute phase following a TBI, defined as 1-7 days posttrauma $[23,25,26]$. Circulating levels of a number of inflammatory cytokines such as interleukin-1 $\beta$ (IL-1 $\beta$ ), interleukin-6 (IL-6), interleukin-8 (IL-8), interleukin-10 (IL$10)$, and tumor necrosis factor- $\alpha$ (TNF- $\alpha$ ) have been examined [16, 18, 27-30]. Only a handful of studies measured the systemic inflammatory response in human populations during the sub-acute phase, roughly 7-30 days or 14 days to 3 months $[23,31,32]$ or later during the chronic period/recovery phase, loosely defined as 3 months to 1 year postTBI [33].
An understanding of the pathophysiology and cytokine response to bTBI comes primarily from experimental blast injury models [34, 35]. Several different experimental animal models of blast-induced TBI, including studies of single blast exposures [36], repeated blast exposures [37], and blast-associated polytrauma [38], have examined inflammatory serum markers. The translation of results, however, from animal models of blast injury to the clinical setting has been problematic. Several challenges have been described including lack of consistency of results across experimental studies, reproducibility, and difficulty in developing experimental models that reflect the conditions of bTBI found in humans. These differences most likely reflect difficulties in determination of local BBB failures and/ or increase in BBB permeability due to mechanical disruption or inflammatory molecules targeting endothelial cells.

Murine studies have shown that inflammatory markers might reflect a variable degree of glymphatic flux and BBB disruption following a TBI [39, 40]. Moreover, glymphatic and meningeal lymphatic systems have been shown to be present in humans [41-43].

There is a paucity of human data on the systemic cytokine immune response during the acute phase $[26,44]$ or during later time points specific for bTBI [45]. Better characterization of the cytokine response in peripheral serum during recovery post-bTBI would help clarify the systemic inflammatory processes during the chronic phase, may provide better monitoring of the recovery or rehabilitative period, and may possibly identify and evaluate therapeutic interventions [46-48]. Clearly, there is a need to understand the changes in circulating cytokines before and after the occurrence of bTBI and how these changes differ from levels among individuals who have not been diagnosed with a bTBI. For these reasons, we conducted a case-control study to examine serum levels of pro- and anti-inflammatory cytokines measured both prior to and during the chronic (recovery) phase after a mild, moderate, or severe bTBI diagnosis, compared with serum levels from pre- and post-deployment/ bTBI among military personnel without a diagnosis of bTBI.

\section{Materials and method Study population}

This longitudinal, case-control study included bTBI cases and controls with a pre-deployment and post-deployment serum sample archived in the Department of Defense (DoD) Serum Repository. This repository houses more than 62 million serum samples from more than 10 million US military service members, dating back to the late 1980s [49]. For the current study, non-penetrating bTBI cases were identified via the Defense Veterans Brain Injury Center (DVBIC) clinical database, a comprehensive database of all TBI patients treated at Walter Reed Army Medical Center between 
February 2003 and August 2010. Identified cases were categorized as mild, moderate, or severe, based on DoD/Veterans Administration (VA) criteria [50]. TBI presence and severity was determined through a clinical assessment involving an interview by a credentialed provider, radiologic findings, review of medical records (including point of injury records when available), and gathering of collateral information (including descriptions by third party observers when available). For cases selected for our study, personal identifiers were encrypted and sent, along with limited affiliated clinical data, from DVBIC to the Armed Forces Health Surveillance Branch (AFHSB) for linkage with DoD Serum Repository samples. Clinical data abstracted for each case included: date of injury, data on severity and nature of extra-cranial injuries (e.g., neck and cervical, face, thorax and thoracic spine, abdomen and lumbar, and extremities), and source of blast injury.

Inclusion criteria for cases were based on age (less than 40 years at the start of first OEF/OIF deployment) and date of injury (after the start of the service member's first OEF/ OIF deployment, but prior to the start of any subsequent deployments). Cases were excluded if they ever had at least one inpatient encounter or two outpatient encounters for any cancer as per the International Classification of Diseases, 9th Edition (ICD-9) codes for neoplasms (codes 140239 ), with the exception of non-melanoma skin cancer. Additionally, exclusions for cases were having had at least one inpatient or two outpatient encounters for schizophrenia (ICD-9 code 295) or bi-polar disorders (ICD-9 codes 296.0, 296.4-.8) any time after the end date of their first OEF/OIF deployment. These exclusions were made because of the potential for cytokine levels to be influenced by carcinogenic processes and psychological conditions. Only cases with a pre-deployment serum specimen collected less than 1 year before the start of their first OEF/OIF deployment and a post-deployment (synonymous with post-bTBI) serum sample were included. The pre- and post-samples meeting these criteria and collected closest to the start and end dates, respectively, of the first OEF/OIF deployment were selected. Since mild cases comprised the majority of our bTBI study population, selection of mild cases was based on preferentially, selecting subjects with the shortest duration between end date of the first OEF/OIF deployment and the date of the post-serum specimen draw date. All moderate bTBI cases that met the inclusion/exclusion criteria were included in the study. Because of the small number of severe bTBI cases, all severe cases identified by the DVBIC database were included in the study population regardless of whether they met the inclusion/exclusion criteria of other pre-existing conditions with the exception that the designated pre- and post-deployment serum specimens were available.
The number of cases identified included 93 mild, 38 moderate, and 19 severe bTBI, for a total of 150 cases. For 1 of the 93 mild cases and 6 of the 19 severe cases, date of postdeployment/bTBI blood draw was found to be erroneous (i.e., prior to date of injury), so these samples were excluded from our analyses. However, the pre-deployment samples for these cases were retained. Given the small number of severe bTBI cases, we combined the moderate and severe cases into one group for analysis (e.g., moderate/severe).

A common set of controls was used for the analysis of mild cases and moderate/severe cases. Eligible controls were required to have had at least one OEF/OIF deployment and be less than 40 years of age at the start of their first OEF/OIF deployment. We excluded from the potential pool of controls any service members listed in the original DVBIC TBI clinical case database or any service member with a medical encounter for intracranial injuries (ICD-9 codes 850-854.19). Additionally, we excluded potential controls who ever had at least one inpatient or two outpatient encounters for the ICD-9 category, neoplasms (ICD-9 codes 140-239), with the exception of non-melanoma skin cancer or at least one inpatient or two outpatient encounters for schizophrenia (ICD-9 code 295) or bi-polar disorders (ICD-9 codes 296.0, 296.4-.8) any time after the end date of their first OEF/ OIF deployment. Criteria for selection of pre-and postsamples used for cases was also applied for controls, resulting in selection of 50 randomly selected controls for the study, based on frequency matching to the case population by age, sex, and race (white, black, other).

Diagnosis of post-traumatic stress disorder (PTSD) was also assessed for each case and control. Two outpatient encounters of ICD-9 code 309.81 in the first diagnostic position, with the first outpatient diagnosis occurring within 4 to 12 months after the end date of the first OEF/OIF deployment and the second diagnosis occurring within 2 years after the end date of the first OEF/OIF deployment, defined PTSD.

\section{Cytokine measurement}

Cytokine analysis was performed on the serum samples using Quantibody Human Cytokine Array 1 platform from Raybiotech, Inc. (Atlanta, GA), which utilizes multiplexed sandwich ELISA-based technology to determine the concentration of multiple cytokines simultaneously.

The Quantibody array was custom made for our study using the following 15 cytokines: nerve growth factor $\beta$ ( $\beta$-NGF), interleukin (IL) $1 \alpha$, IL1 $\beta$, IL4, IL6, IL8, IL10, IL13, IL17, monocyte chemoattractant protein-1 (MCP1), matrix metallopeptidase 3 (MMP-3), matrix metallopeptidase 9 (MMP-9), transforming growth factor $\beta-1$ (TGF $\beta-1)$, TNF $\alpha$, and TNF $\beta$. At the time of experiment, slides were equilibrated to room temperature for 20-30 min and air dried for another $1-2 \mathrm{~h}$. 
A standard glass slide array was spotted with 16 wells of identical cytokine antibody. In this study, $30 \mu \mathrm{l}$ of serum, together with positive and negative controls, were arrayed in quadruplicate. Standard cytokines and samples were assayed on each array simultaneously. The lyophilized cytokine standards were reconstituted and serial dilutions prepared using the sample diluent. The concentration of this cytokine standard was predetermined and provided along with the array kit to generate a standard curve in order to determine the concentration of each cytokine in the experimental sample. Sample diluent alone served as a negative control. The blocking of the slide was done by adding $100 \mu \mathrm{l}$ of the sample diluent into each well of the glass slide and incubated for $30 \mathrm{~min}$ at room temperature to block the slides. The blocking buffer was decanted. The standard cytokines of eight different dilutions of samples of $100 \mu \mathrm{l}$ volume were added into each well and the arrays incubated at $4{ }^{\circ} \mathrm{C}$ overnight with constant shaking. The following day, samples were decanted and wells were washed five times with $150 \mu \mathrm{l}$ of $1 \times$ wash buffer I and two times with wash buffer II for 5 min each wash with gentle shaking at room temperature. Then, $80 \mu \mathrm{l}$ of diluted detection antibody was added to each well and incubated at room temperature for 1-2 $\mathrm{h}$ with gentle shaking followed by washing with wash buffers I and II as before. Following this, $80 \mu \mathrm{l}$ of $\mathrm{Cy} 3$ equivalent dye-conjugated streptavidin was added to each well and the slides were covered with foil and incubated for $1 \mathrm{~h}$ at room temperature with shaking. Wells were then washed with $150 \mu \mathrm{l}$ of wash buffer I for five times at room temperature with gentle shaking. After the last wash, the slide was disassembled, removed from the gasket, and placed in the slide holder containing wash buffer1. The slides were incubated for $15 \mathrm{~min}$ and then washed with wash buffer 2, air-dried, and stored in dark at $4{ }^{\circ} \mathrm{C}$. The slides were imaged with a laser scanner equipped with a $\mathrm{Cy} 3$ wavelength such as Axon GenePix, and data extraction was performed by Raybiotech, using microarray Quantibody Q-Analyzer data computation software for quantitative data analysis. The concentration of each cytokine in a sample was measured by comparing signals of unknown samples to the standard curve to determine the concentration of cytokine in each sample, and the final results were provided as $\mathrm{pg} / \mathrm{ml}$ for further analysis.

A small number of samples were not measureable (i.e., three post-deployment/bTBI samples from controls) and some samples had readings above the maximum detectable level (i.e., one mild case pre-deployment, two mild cases post-deployment/bTBI, and one moderate case predeployment). These samples were, therefore, excluded from further analyses. Additionally, the array did not provide results for TNF $\beta$ (all zero values), and the majority of readings for MMP-9 were below the detection limit, precluding us from evaluating these markers. Samples were randomly ordered on plates by case/control status, and all laboratory personnel were blinded to case status. Intra-assay coefficients of variation $(\mathrm{CV})$ were calculated for each slide, based on duplicate positive controls, and the maximum $\mathrm{CV}$ detected was $7 \%$.

\section{Statistical analyses}

As mentioned above, given the small number of severe bTBI cases, we conducted analyses for moderate and severe cases combined. Using paired $t$ tests, we first compared pre- and post-deployment/bTBI mean cytokine levels among cases and among controls. Using generalized linear models (GLM), we determined mild bTBI case-control differences in mean cytokine levels, pre-deployment and post-deployment/bTBI, with adjustment for age, the only demographic factor that significantly differed between mild cases and controls. As no demographic factors differed between moderate/severe cases and controls, we used a simple $t$ test for comparisons of mean cytokine levels between these cases and controls. Finally, we estimated the mean change in pre-post deployment cytokine levels for cases versus the change in pre-post cytokine levels for controls, using analysis of variance (ANOVA), adjusting for age in the comparisons including mild cases. We also conducted analyses stratified by age $(<25, \geq 25$ years), length of deployment $(\leq 215,>215$ days (median length of deployment)), extracranial injury (with, without), PTSD (with, without), and time between injury and serum sample collection for various time cut-points (<3 months, $\geq 3$ months; < 6 months, $\geq 6$ months; < 252 days, $\geq 252$ days (median); $<365$ days, $\geq 365$ days). Because of the study sample size, we were unable to stratify by more lag time periods between injury and serum sample collection than two at a given time. We carried out sensitivity analyses to evaluate if our findings persisted after excluding cases with a PTSD diagnosis and after excluding cases without an extra-cranial injury.

Given the skewed distribution of the serum cytokine data, we evaluated analyses using log-transformed cytokine measures. Since the final results using the original, non-log transformed cytokine measurements were similar to the log-transformed data, we present the nontransformed data results in the paper.

SAS procedures $t$ test, GLM, and ANOVA were used for the data analysis (SAS Version 9.3) [51]. This study was approved by the Institutional Review Boards at the Uniformed Services University of the Health Sciences and the Walter Reed Army Medical Center.

\section{Results}

\section{Characteristics of cases and controls}

The final sample sizes, after consideration of the noted exclusions, included 91 mild cases, 37 moderate cases, 19 severe cases, for a total of 147 cases and 50 controls for the analysis of pre-deployment samples, and 90 mild cases, 38 moderate cases, 13 severe cases, for a total of 
141 cases and 47 controls for analyses of the postdeployment/bTBI samples. Baseline characteristics of the study population are presented in Table 1. All cases combined and controls did not differ by age, gender, and race, since controls were frequency matched to cases on these demographic factors. When we evaluated baseline characteristics for the two case groups (mild; moderate/ severe) separately, however, mild bTBI cases were statistically significantly younger than controls, but there were no statistically significant age differences between moderate/ severe cases and controls. No controls had a diagnosis of PTSD, while about $25 \%$ of cases had a PTSD diagnosis based on ICD-9 coded health encounter data. Most mild cases (85\%) had some type of extra-cranial injury in addition to a traumatic brain injury. No significant differences existed between the two case groups and controls with respect to time between pre-deployment serum collection and deployment start date or length of deployment; the average time between injury onset and post-serum draw was $315.8(\mathrm{sd}=261.1)$ days among all cases post-deployment/bTBI, $281.8(\mathrm{sd}=$ 240.3) days for mild cases, and $377.0(\mathrm{sd}=287.4)$ days among moderate/severe cases. Among controls, the mean time between end of deployment and serum draw postdeployment was $192.4(\mathrm{sd}=280.2)$ days.

\section{Pre- and post-deployment comparisons}

Table 2 compares pre-deployment and post-deployment/ bTBI mean cytokine levels separately for controls, mild cases, and combined moderate/severe cases, using paired $t$ tests. The IL-8 cytokine levels declined from pre- to postserum samples for both mild cases (pre, $155.34 \mathrm{pg} / \mathrm{ml}$; post, $71.92 \mathrm{pg} / \mathrm{ml} ; p<0.01$ ) and for moderate/severe cases (pre, $186.01 \mathrm{pg} / \mathrm{ml}$; post, $78.34 \mathrm{pg} / \mathrm{ml} ; \mathrm{p}<0.01$ ). Among moderate/severe cases, TGF $\beta 1$ cytokine levels also declined from pre- to post- for moderate/severe cases (pre, $4739.94 \mathrm{pg} / \mathrm{ml} ;$ post, $2873.00 \mathrm{pg} / \mathrm{ml} ; p=0.05$ ). Similar declining patterns, albeit non-statistically significant, were found among moderate/severe cases for IL-6 (pre, 52.10 $\mathrm{pg} / \mathrm{ml}$; post, $20.50 \mathrm{pg} / \mathrm{ml} ; p=0.08$ ) and for MMP3 (pre, 21 , $679.6 \mathrm{pg} / \mathrm{ml}$; post, $18,310.36 \mathrm{pg} / \mathrm{ml} ; p=0.07$ ), pre- to postThere were no statistically significant differences from pre- to post-deployment for controls, although IL-8 levels tended to be higher in controls' post-deployment serum samples.

\section{Case-control comparisons of cytokine change, pre- to post-deployment}

Table 3 shows the pre- and post-deployment/bTBI mean differences in cytokine levels for cases versus controls.

Table 1 Baseline characteristics of study population of cases and controls

\begin{tabular}{|c|c|c|c|c|c|c|c|c|c|c|c|}
\hline & \multicolumn{2}{|c|}{ All Cases } & \multicolumn{2}{|c|}{ Controls } & \multirow[t]{2}{*}{$p\left(x^{2}\right)$} & \multicolumn{2}{|c|}{ Mild TBI Cases } & \multirow[t]{2}{*}{$p\left(x^{2}\right)^{a}$} & \multicolumn{2}{|c|}{$\begin{array}{c}\text { Moderate + } \\
\text { SevereTBI Cases }\end{array}$} & \multirow[t]{2}{*}{$p\left(x^{2}\right)^{b}$} \\
\hline & $N=150$ & $\%$ & $N=50$ & $\%$ & & $\mathrm{~N}=93$ & $\%$ & & $\mathrm{~N}=57$ & $\%$ & \\
\hline \multicolumn{12}{|l|}{ Categorical Characteristics } \\
\hline \multicolumn{12}{|l|}{ Age } \\
\hline$<20$ & 20 & (13.3) & 3 & $(6.0)$ & \multirow{4}{*}{0.19} & 14 & (15.1) & \multirow{4}{*}{$<0.01$} & 6 & (10.5) & \multirow{4}{*}{0.67} \\
\hline $20-24$ & 82 & $(54.7)$ & 27 & $(54.0)$ & & 56 & $(60.2)$ & & 26 & $(45.6)$ & \\
\hline $25-29$ & 33 & $(22.0)$ & 10 & $(20.0)$ & & 23 & $(24.7)$ & & 10 & $(17.5)$ & \\
\hline$>=30$ & 15 & $(10.0)$ & 10 & $(20.0)$ & & 0 & $(0.0)$ & & 15 & $(26.3)$ & \\
\hline \multicolumn{12}{|l|}{ Gender } \\
\hline Male & 144 & $(96.0)$ & 48 & $(96.0)$ & \multirow{2}{*}{1.00} & 89 & (95.7) & \multirow{2}{*}{0.93} & 55 & (96.5) & \multirow{2}{*}{0.89} \\
\hline Female & 6 & $(4.0)$ & 2 & $(4.0)$ & & 4 & $(4.3)$ & & 2 & $(3.5)$ & \\
\hline \multicolumn{12}{|l|}{ Race } \\
\hline White & 129 & $(86.0)$ & 43 & $(86.0)$ & \multirow{3}{*}{0.98} & 81 & $(87.1)$ & \multirow{3}{*}{0.94} & 48 & $(84.2)$ & \multirow{3}{*}{0.97} \\
\hline Black & 10 & $(6.7)$ & 3 & $(6.0)$ & & 6 & $(6.5)$ & & 4 & $(7.0)$ & \\
\hline Other & 11 & (7.3) & 4 & $(8.0)$ & & 6 & (6.5) & & 5 & (8.8) & \\
\hline \multicolumn{12}{|l|}{ Deployment Length } \\
\hline Shorter (<12 months) & 122 & $(81.3)$ & 42 & $(84.0)$ & \multirow{2}{*}{0.67} & 79 & $(85.0)$ & \multirow{2}{*}{0.88} & 43 & $(75.4)$ & \multirow{2}{*}{0.27} \\
\hline Longer (>=12 months) & 28 & $(18.7)$ & 8 & $(16.0)$ & & 14 & $(15.1)$ & & 14 & $(24.6)$ & \\
\hline \multicolumn{12}{|l|}{ PTSD } \\
\hline No & 111 & $(75.0)$ & 42 & $(100.0)$ & & 67 & (73.6) & \multirow{3}{*}{0.00} & 44.00 & $(77.2)$ & \multirow{3}{*}{0.00} \\
\hline Yes & 37 & (25.0) & 0 & $(0.0)$ & $<0.01$ & 24 & (26.4) & & 13.00 & (22.8) & \\
\hline Missing & 2 & & 8 & & & & & & & & \\
\hline Extra Cranial Injury & & & & & & & & & & & \\
\hline No & 15 & $(10.0)$ & - & & & 13 & $(14.0)$ & & 2 & $(3.5)$ & \\
\hline Yes & 135 & $(90.0)$ & - & & & 80 & $(86.0)$ & & 55 & (96.5) & \\
\hline Continuous Characteristics & Mean & Std & Mean & Std & $p$ (t-test) & Mean & Std & T-test P & Mean & Std & $p$ (t-test) \\
\hline Length of Deployment (days) & 248.0 & (171.5) & 219.2 & $(125.8)$ & 0.28 & 251.3 & (185.1) & 0.28 & 242.7 & (148.1) & 0.38 \\
\hline Time from Pre-Deployment Serum to Deployment Start (days) & -98.7 & (78.8) & -117.8 & (93.5) & 0.16 & -94.0 & (75.0) & 0.10 & -106.3 & (84.9) & 0.51 \\
\hline Time from Injury to Post-Deployment Serum (days) - Cases & 315.8 & (261.1) & & & & 281.8 & (240.3) & & 377.0 & (287.4) & \\
\hline Time from Deplyoment End to Post-Deployment Serum (days) - Controls & & & 192.4 & $(280.2)$ & & & & & & & \\
\hline a $\mathrm{p}$-value for comparison of mild cases to controls & & & & & & & & & & & \\
\hline${ }^{\mathrm{b}} \mathrm{p}$-value for comparison of moderate/severe cases to controls & & & & & & & & & & & \\
\hline
\end{tabular}


Table 2 Comparisons of mean cytokine levels pre- and post-deployment/bTBI for controls, mild cases, and moderate/severe cases, via paired t-test

\begin{tabular}{|c|c|c|c|c|c|c|c|c|c|c|c|c|c|}
\hline \multirow{2}{*}{$\begin{array}{l}\text { Gene/ } \\
\text { locus }\end{array}$} & \multirow[t]{2}{*}{ Comparison } & \multicolumn{4}{|c|}{ Control } & \multicolumn{4}{|c|}{ Mild } & \multicolumn{4}{|c|}{ Moderate+Severe } \\
\hline & & $\mathrm{N}$ & mean & s.e. & $p$-value & $\mathrm{N}$ & mean & s.e. & $p$-value & $\mathrm{N}$ & mean & s.e. & $p$-value \\
\hline \multirow[t]{2}{*}{$\beta N G F$} & Pre & 47 & 89.72 & 22.39 & & 89 & 70.45 & 18.64 & & 50 & 63.54 & 10.69 & \\
\hline & Post & & 50.57 & 9.35 & 0.10 & & 66.00 & 15.98 & 0.84 & & 56.14 & 14.28 & 0.70 \\
\hline \multirow[t]{2}{*}{ IL-1a } & Pre & 47 & 15.61 & 3.34 & & 89 & 29.65 & 5.95 & & 50 & 29.41 & 6.54 & \\
\hline & Post & & 23.77 & 4.88 & 0.11 & & 21.33 & 4.39 & 0.20 & & 19.49 & 3.47 & 0.18 \\
\hline \multirow[t]{2}{*}{ IL-1 $\beta$} & Pre & 47 & 11.71 & 4.77 & & 89 & 15.87 & 5.97 & & 50 & 13.94 & 4.86 & \\
\hline & Post & & 13.27 & 4.74 & 0.81 & & 6.05 & 1.61 & 0.12 & & 5.96 & 1.97 & 0.15 \\
\hline \multirow[t]{2}{*}{ IL-4 } & Pre & 47 & 4.91 & 0.88 & & 89 & 5.22 & 1.04 & & 50 & 5.67 & 1.34 & \\
\hline & Post & & 7.42 & 1.60 & 0.15 & & 4.72 & 0.98 & 0.65 & & 4.07 & 0.96 & 0.25 \\
\hline \multirow[t]{2}{*}{ IL-6 } & Pre & 47 & 39.69 & 14.86 & & 89 & 34.76 & 9.19 & & 50 & 52.10 & 16.69 & \\
\hline & Post & & 44.82 & 9.75 & 0.77 & & 24.94 & 3.95 & 0.32 & & 20.50 & 5.44 & 0.08 \\
\hline \multirow[t]{2}{*}{ |L-8 } & Pre & 45 & 95.04 & 22.78 & & 88 & 155.34 & 18.99 & & 50 & 186.01 & 29.72 & \\
\hline & Post & & 127.90 & 26.69 & 0.28 & & 71.92 & 11.66 & $<0.01$ & & 78.34 & 17.08 & $<0.01$ \\
\hline \multirow[t]{2}{*}{ IL-10 } & Pre & 47 & 10.62 & 1.77 & & 89 & 10.17 & 1.86 & & 50 & 9.64 & 1.28 & \\
\hline & Post & & 9.38 & 1.85 & 0.58 & & 10.08 & 1.81 & 0.95 & & 10.00 & 1.94 & 0.87 \\
\hline \multirow[t]{2}{*}{ IL-13 } & Pre & 47 & 65.04 & 12.11 & & 89 & 64.09 & 13.72 & & 50 & 74.76 & 13.06 & \\
\hline & Post & & 54.35 & 11.91 & 0.43 & & 56.56 & 13.08 & 0.44 & & 49.74 & 8.79 & 0.12 \\
\hline \multirow[t]{2}{*}{ IL-17 } & Pre & 47 & 63.89 & 10.84 & & 89 & 66.67 & 10.85 & & 50 & 78.87 & 11.47 & \\
\hline & Post & & 78.76 & 19.05 & 0.45 & & 81.13 & 16.26 & 0.27 & & 63.12 & 11.78 & 0.29 \\
\hline \multirow[t]{2}{*}{ MCP1 } & Pre & 47 & 105.45 & 8.73 & & 89 & 120.95 & 7.34 & & 50 & 111.17 & 6.94 & \\
\hline & Post & & 116.17 & 8.30 & 0.40 & & 128.71 & 9.98 & 0.54 & & 107.22 & 8.99 & 0.70 \\
\hline \multirow[t]{2}{*}{ MMP3 } & Pre & 47 & $20,732.20$ & $1,297.38$ & & 85 & $21,995.44$ & 966.14 & & 49 & $21,679.60$ & $1,144.74$ & \\
\hline & Post & & $22,591.80$ & $1,428.18$ & 0.33 & & $20,043.55$ & 971.06 & 0.10 & & $18,310.36$ & $1,235.37$ & 0.07 \\
\hline \multirow[t]{2}{*}{ TGF $\beta 1$} & Pre & 47 & $5,097.67$ & 999.86 & & 89 & $5,378.17$ & 943.02 & & 50 & $4,739.94$ & 774.48 & \\
\hline & Post & & $4,649.21$ & $1,279.54$ & 0.79 & & $4,663.20$ & $1,163.21$ & 0.50 & & $2,873.00$ & 647.87 & 0.05 \\
\hline \multirow[t]{2}{*}{ TNFa } & Pre & 47 & 682.54 & 96.21 & & 89 & 644.05 & 85.58 & & 50 & 702.38 & 107.64 & \\
\hline & Post & & 690.78 & 127.97 & 0.96 & & 706.96 & 89.89 & 0.59 & & 528.07 & 84.81 & 0.20 \\
\hline
\end{tabular}

Consistent with findings presented in Table 2, mean serum levels of IL-8 decreased pre- to post-deployment/bTBI for mild cases $(-83.43)$ and moderate/severe cases $(-107.67)$, but increased slightly among controls $(+32,86), p<0.01$ for both comparisons. The only other statistically significant difference was found for MMP3 levels among moderate/severe cases $(-3369.24)$ versus controls $(+1859.60), p=0.03$; a similar, non-statistically significant pattern was found among mild cases. Marginally significant case-control differences were also found for IL- $1 \alpha$ and IL- 4 in both mild cases and moderate/severe cases and for IL-6 among moderate/severe cases. Taken together, these results showed a similar pattern of decreasing cytokine levels among cases and marginally increasing levels among controls, comparing pre- to post-deployment/bTBI samples. These findings are further elucidated in Additional file 1: Table S1, which presents case-control comparisons both pre- and postdeployment/bTBI. Here, we show that post-deployment/
bTBI controls' cytokine levels were greater than cases' for IL-6 $\left(p_{\text {mild }}=0.02 ; p_{\text {moderate } / \text { severe }}=0.01\right), \mathrm{IL}-8 \quad\left(p_{\text {mild }}=0.01\right.$; $\left.p_{\text {moderate } / \text { severe }}=0.04\right)$, and MMP3 $\left(p_{\text {moderate } / \text { severe }}=0.03\right)$. An additional significant difference found was that postdeployment/bTBI controls' cytokine levels were greater than cases' for IL-1 $\beta$ ( $\left.p_{\text {mild }}=0.05 ; p_{\text {moderate } / \text { severe }}=0.09\right)$. It should be noted that pre-deployment cases had higher levels than controls for IL-8 ( $p_{\text {mild }}=0.05 ; p_{\text {moderate }}$ severe $=$ 0.01). All other comparisons pre-deployment showed no difference between cases and controls.

\section{IL-8 further analysis}

We further investigated findings for IL- 8 by evaluating IL-8 pre- and post-deployment/bTBI mean differences for cases versus controls, stratified by age, length of deployment, evidence of extra-cranial injury, PTSD diagnosis, and time between injury and blood sample draw (Additional file 1: Table S2). Analyses stratified by age 
Table 3 Results of ANOVA with Single Model comparing means of pre-post cytokine difference for cases versus controls

\begin{tabular}{|c|c|c|c|c|c|c|c|c|c|}
\hline \multirow[t]{2}{*}{ Gene } & \multirow[t]{2}{*}{ Comparison } & \multicolumn{4}{|c|}{ Mild bTBI Cases ${ }^{a}$} & \multicolumn{4}{|c|}{ Moderate+Severe bTBI Cases } \\
\hline & & $\mathrm{N}$ & mean & (s.e.) & $p$-value & $\mathrm{N}$ & mean & (s.e.) & $p$-value \\
\hline \multirow[t]{2}{*}{$\beta N G F$} & Case & 89 & -4.44 & 19.24 & & 50 & -7.39 & 25.67 & \\
\hline & Control & 47 & -39.15 & 26.47 & 0.29 & 47 & -39.15 & 26.47 & 0.39 \\
\hline \multirow[t]{2}{*}{ IL-1a } & Case & 89 & -8.33 & 5.58 & & 50 & -9.92 & 7.45 & \\
\hline & Control & 47 & 8.16 & 7.68 & 0.08 & 47 & 8.16 & 7.68 & 0.09 \\
\hline \multirow[t]{2}{*}{$\mid L-1 \beta$} & Case & 89 & -9.81 & 5.33 & & 50 & -7.98 & 7.11 & \\
\hline & Control & 47 & 1.56 & 7.33 & 0.21 & 47 & 1.56 & 7.33 & 0.35 \\
\hline \multirow[t]{2}{*}{ |L-4 } & Case & 89 & -0.50 & 1.11 & & 50 & -1.60 & 1.49 & \\
\hline & Control & 47 & 2.51 & 1.53 & 0.11 & 47 & 2.51 & 1.53 & 0.06 \\
\hline \multirow[t]{2}{*}{ IL-6 } & Case & 89 & -9.82 & 11.62 & & 50 & -31.60 & 15.51 & \\
\hline & Control & 47 & 5.13 & 15.99 & 0.45 & 47 & 5.13 & 15.99 & 0.10 \\
\hline \multirow[t]{2}{*}{ |L-8 } & Case & 88 & -83.43 & 21.66 & & 50 & -107.67 & 28.74 & \\
\hline & Control & 45 & 32.86 & 30.29 & $<0.01$ & 45 & 32.86 & 30.29 & $<0.01$ \\
\hline \multirow[t]{2}{*}{ |L-10 } & Case & 89 & -0.10 & 1.52 & & 50 & 0.36 & 2.03 & \\
\hline & Control & 47 & -1.24 & 2.09 & 0.66 & 47 & -1.24 & 2.09 & 0.58 \\
\hline \multirow[t]{2}{*}{ IL-13 } & Case & 89 & -7.54 & 10.38 & & 50 & -25.03 & 13.85 & \\
\hline & Control & 47 & -10.69 & 14.28 & 0.86 & 47 & -10.69 & 14.28 & 0.47 \\
\hline \multirow[t]{2}{*}{ |L-17 } & Case & 89 & 14.46 & 12.88 & & 50 & -15.76 & 17.19 & \\
\hline & Control & 47 & 14.87 & 17.73 & 0.99 & 47 & 14.87 & 17.73 & 0.22 \\
\hline \multirow[t]{2}{*}{ MCP1 } & Case & 89 & 7.76 & 10.69 & & 50 & -3.95 & 14.26 & \\
\hline & Control & 47 & 10.72 & 14.71 & 0.87 & 47 & 10.72 & 14.71 & 0.47 \\
\hline \multirow[t]{2}{*}{ MMP3 } & Case & 85 & $-1,951.88$ & $1,292.01$ & & 49 & $-3,369.24$ & $1,701.68$ & \\
\hline & Control & 47 & $1,859.60$ & $1,737.51$ & 0.08 & 47 & $1,859.60$ & $1,737.51$ & 0.03 \\
\hline \multirow[t]{2}{*}{ TGF $\beta 1$} & Case & 89 & -714.97 & $1,006.08$ & & 50 & $-1,866.94$ & $1,342.28$ & \\
\hline & Control & 47 & -448.46 & $1,384.46$ & 0.88 & 47 & -448.46 & $1,384.46$ & 0.46 \\
\hline \multirow[t]{2}{*}{ TNFa } & Case & 89 & 62.92 & 109.85 & & 50 & -174.31 & 146.55 & \\
\hline & Control & 47 & 8.25 & 151.16 & 0.77 & 47 & 8.25 & 151.16 & 0.39 \\
\hline
\end{tabular}

${ }^{\text {a Adjusted for age }}$

groups showed that the decreases for both mild and moderate/severe cases post-injury and increase for controls postdeployment were more pronounced for subjects younger than 25 years of age (mild cases $-85.87 \mathrm{pg} / \mathrm{ml}$, controls + $41.46 \mathrm{pg} / \mathrm{ml}, p=0.01$; moderate $/$ severe cases $-161.38 \mathrm{pg} / \mathrm{ml}$, controls $+41.46 \mathrm{pg} / \mathrm{ml}, p=0.01$ ). For both mild cases and moderate/severe cases, mean differences were more pronounced for those with shorter ( $\leq 215$ days) deployments (mild cases -108.35 , controls 78.92, $p<0.01$; moderate/severe cases -116.08 , controls $78.92, p<0.01$ ). Additionally, the decreases for both mild and moderate/severe cases postinjury appeared to be more pronounced for cases with PTSD. There were no apparent differences in mean levels for those with an extra-cranial injury and without. For the lag time between bTBI diagnosis and post-deployment/bTBI serum collection, we saw a pattern of mild cases with a shorter time interval (i.e., $<3$ months) having a greater reduction in cytokine levels than those with a longer time interval
( $\geq 3$ months). We also noted similar patterns for other stratifications of time between injury and serum collection (i.e., for $<6$ months vs. $\geq 6$ months, etc.). For the longer lag periods between injury and serum collection (i.e., 252, 365 days), the difference between cases' pre-/post-changes and controls' pre-/post-changes was reduced, and by 365 days, the case/control difference was no longer statistically significant. For moderate/severe cases, there were still statistically significant differences between cases and controls beyond 365 days.

Figure 1 presents mean levels of IL- 8 among all cases and among mild cases at various lag times between bTBI diagnosis and post-deployment/bTBI serum collection: $\leq 90$ days, 90 to $<180$ days, 180 to $<365$ days, and $\geq 365$ days. Each bar represents different groups of cases, thus not an illustration of serial measures, rather a presentation of the levels of cases within each of these lag time categories. In general, post-deployment/bTBI IL-8 levels 


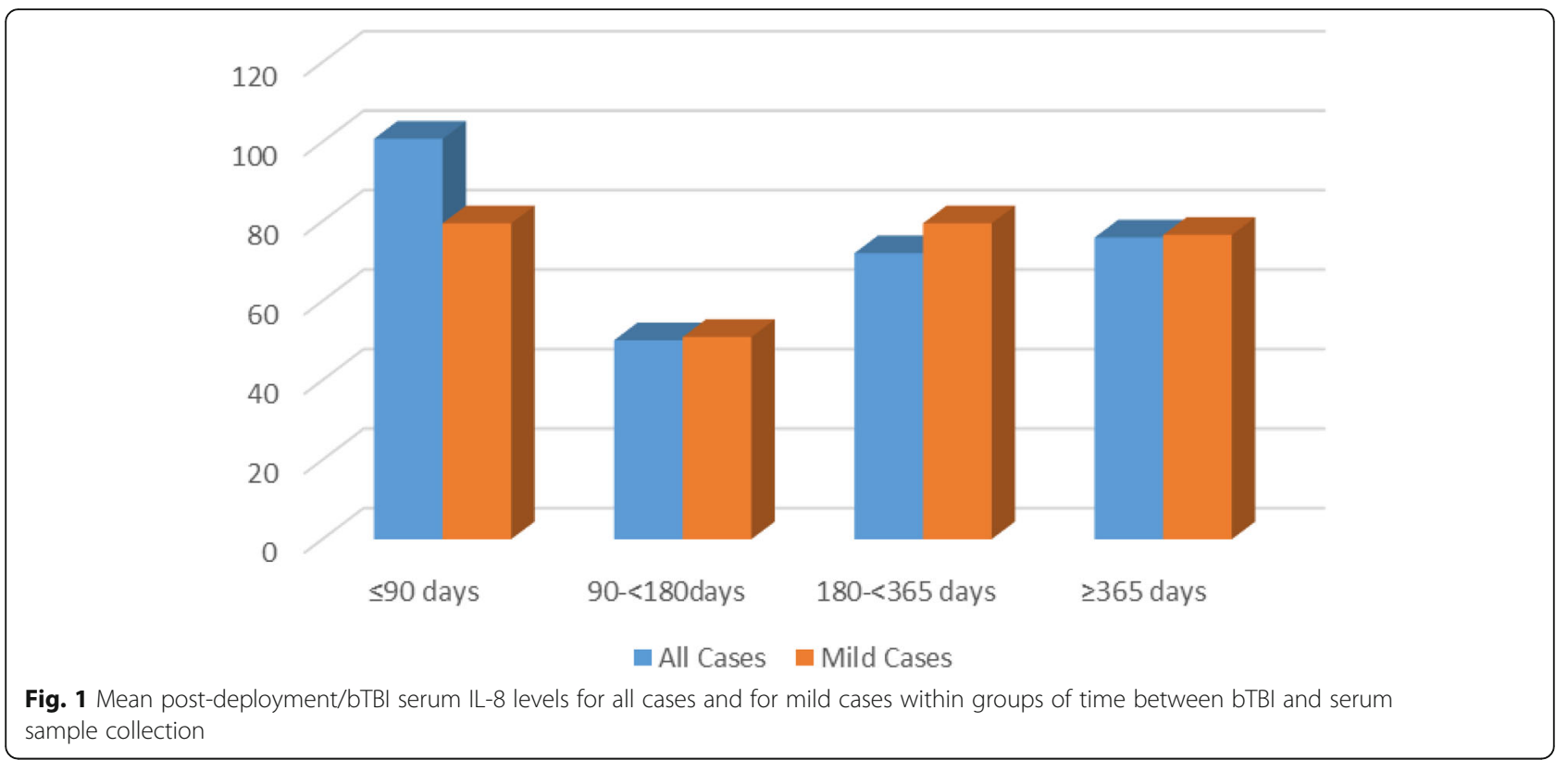

appear to be quite stable over time for all cases and specifically for mild cases. We do not present results for moderate/severe cases, since numbers were small after stratifying into these four groups.

\section{Discussion}

In this longitudinal study, we found that circulating serum levels of IL-8 significantly decreased from pre- to post-deployment/bTBI for both mild and moderate/severe bTBI cases. We also found that post-deployment/ bTBI, both mild and moderate/severe cases, had lower IL-8 levels than controls and that the change in levels pre- to post- were significantly different between cases and controls, being more pronounced for moderate/severe cases, a difference driven more strongly by the decline in cases' levels than the increase in controls' levels. Intriguingly, we found that pre-deployment, controls' IL8 levels were significantly lower than both mild and moderate/severe cases' levels, though controls' levels did not significantly change from pre- to post-deployment. Decreased IL-8 serum levels were more pronounced among younger mild and mod/severe bTBI cases, those with shorter deployments, and those with PTSD. It is unclear why these sub-groups had more pronounced decreases in IL-8, and this should potentially be explored in future studies with larger sample sizes in these groups. With increasing lag time between injury and serum measurement, the pre-/post-difference between cases and controls was reduced for mild cases but remained significantly different for moderate/severe cases. We also observed a statistically significant reduction in MMP3 levels among moderate/severe bTBI cases compared with controls in pre- versus post-deployment/
bTBI samples. Although differences were not statistically significant at $\alpha \leq 0.05$, similar patterns were found for IL- $1 \alpha$, IL-4, and IL- 6 . To our knowledge, this is the first human study focused on bTBI which includes both preand long-term post-injury cytokine measures.

The peripheral inflammatory response that is activated in response to injury is mediated by the complex release of cytokines which facilitate and inhibit inflammatory responses $[18,21,52]$. IL- $\beta$ is a pro-inflammatory cytokine whose involvement in neuroinflammation during the acute phase post-injury has been well-described. IL-8 is a chemokine also known as CXCL8 that recruits inflammatory cells to the site of injury. IL-4 is considered an anti-inflammatory cytokine that can downregulate the production of other proinflammatory cytokines. IL-6 has been extensively studied in TBI, albeit in the acute phase, and is considered to be a singular marker of inflammation [53]. MMPs, and in particular, MMP3 and MMP9, are upregulated during TBI, because of $\mathrm{BBB}$ disruption, and are involved in neuroinflammation and cell death [54]. It is unclear, however, whether inflammation persists over time as measured by serum cytokine levels in the setting of TBI or bTBI [52].

While some studies have examined peripheral cytokine levels soon after bTBI $[26,44]$, very limited data are available to compare with our study results that describe the systemic inflammatory response during the chronic/recovery phase post-bTBI or even post-TBI. Kumar and colleagues [33] examined several inflammatory cytokines with serum specimens obtained at 2-week intervals for the first 6 months and at 12 months post-injury among severe TBI cases and controls. Several combinations of cytokines were analyzed and in conjunction with poor outcomes at 6 months and 12 months, as measured by the Glasgow 
Outcome Scale (GOS) scores. Overall, elevated levels of IL$1 \beta$, IL-6, IL-8, and TNF- $\alpha$ were seen over a 3-month period following severe TBI; however, that study did not include pre-injury serum cytokines levels, thus precluding comparison to pre-injury levels. Interestingly, the study also showed that levels of IL-8 increased among cases from 6 months to 12 months. Devoto and colleagues reported elevated serum levels of IL-6, IL-10, and TNF- $\alpha$ among bTBI cases compared with controls during the recovery period, post-injury [45]. Serum samples were obtained within 16 months after deployment, but the timing of the specimen collection during recovery phase in relation to the bTBI diagnosis was not described. A recent study evaluated the changes in cytokine concentrations pre- and post- a blast-related training program, finding that those exposed to higher blast pressures had higher cytokine (IL-6) concentrations but that those concentrations rebounded to baseline levels the day after the training blast exposure [26].

The idea that elevated serum levels of inflammatory cytokines might persist during the chronic phase following a bTBI originates from studies showing that elevated cytokines levels measured during the acute phase post-TBI were predictive of poor outcomes up to 1 year post-injury, as measured by the GOS scores $[33,55]$. The results presented here are not in agreement with these studies. However, the average time between injury and post-injury serum draw among cases in our study was approximately 10.5 months (315.8 days), and relatively few samples from cases in our study were among those with less than 1 month between injury and post-injury serum draw (8 mild cases; 5 moderate/severe cases).

The attenuation in bTBI cases of pro-inflammatory cytokines as IL-6, IL- 8 and IL1- $\alpha$ found in our study may suggest an ongoing neuroprotective mechanism related to the suppression of NF-kB activation and/or inhibition of p38 mitogen-activated protein kinase (MAPK) $[56,57]$. Regarding the attenuation of MMP3, this cytokine belongs to a large family of endoproteinases primarily involved in turnover and remodeling of the extracellular matrix, and its expression in the brain is largely restricted to astrocytes [17], possibly impacting astrocyte interactions with blood vessels with astroglial scaring, considered as the hallmark of blast injury [14]. Changes in peripheral blood composition due to a post-blast chronic progressive or healing/neuroprotective condition may also affect the detected cytokine levels, as previously reported [58-60]. The cytokine stability in serum may be affected by storage conditions [61, 62], but according to one study [63], freeze thaw cycles should not significantly affect levels of some of the cytokines measured in our study (TNF- $\alpha$ and IL-6). The results reported here suggest that long-term cytokine profiles may not reflect changes seen immediately following blast results; however, we cannot completely exclude comorbidity with other clinical conditions and we cannot rule out the influence of drug therapy influencing these levels.
This study has several strengths and limitations which should be pointed out. We had unique access to serum samples, both prior to bTBI and prior to OEF/ OIF deployment and post-deployment/bTBI, which allowed comparisons of baseline levels of circulating cytokine levels with serum levels determined after an initial bTBI diagnosis. Other strengths of this study are the relatively large sample size of 150 cases of mild and moderate/severe bTBI identified from clinical records and the ability to measure cytokine levels during the recovery period as serum samples were identified over a 12-month period following a bTBI diagnosis. Paired analyses revealed that controls' levels did not differ significantly between pre- and post-deployment. It is important to include a control group in this type of study, and this control group likely represents the cohort which gave rise to these cases, given both cases and controls are from the active duty military with OEF/OIF deployment. The serum samples were handled using standard laboratory procedures, assayed on the same ELISA plate, with laboratory personnel blinded to case-control status. Of note, the CV\% for each cytokine was low, in the range of $7 \%$ or less. Limitations include the possibility that some unaccountable differences exist between the bTBI cases and controls, in addition to brain injury, that affected the cytokine levels to produce these observed results. Of note, we also did not have access to information on treatment modalities for the mild or moderate/severe bTBI cases, the effects of which could have altered the detected cytokine levels in the post-deployment/bTBI serum samples. However, some of the most commonly used treatment interventions in TBI, such as psychostimulants, antidepressants, and anticonvulsants, operate via binding to dopamine transporters, increasing dopamine levels in the brain, affecting levels of norepinephrine and serotonin or enhancing inhibitory control mediated by neurotransmitters [64]. It is unclear if inflammatory cytokine levels are influenced by these common treatments; however, this should be considered in future studies. Headache, a common symptom in TBI patients is sometimes treated with nonsteroidal anti-inflammatory or triptans (5-HT agonists). The extent to which that was a factor in the current study is unclear. Similarly, as described above, sleep disturbance, common in TBI patients, may affect the cerebral spinal fluid (CSF) and lymphatic/glymphatic clearance and, as shown in murine models, may reduce cytokine levels in serum following a TBI $[65,66]$. However, our study did not include data on sleep disturbance. Increased inflammatory cytokines and MMPs have been implicated in migraine, and we also did not have data in our study about migraine. These factors could significantly impact the outcome of our analysis. In addition, a PTSD diagnosis was 
determined from medical encounter databases rather than from clinical records, resulting in the possibility of some misclassification of the PTSD diagnosis. Finally, given our small sample size, stratification by lag time between injury and serum sample was limited.

\section{Conclusions}

In conclusion, we found an indication that circulating cytokine levels in mild and moderate/severe bTBI cases differed from controls during the recovery phase after bTBI in pre-to post-deployment/bTBI serum samples, with IL-8 levels decreased in mild and moderate/severe bTBI and MMP3 levels decreased in moderate/severe bTBI. There was limited evidence of reduced serum levels of IL-1a, IL-4, and IL-6 during the recovery period. Our study results represent the chronic stage post-bTBI and should be interpreted as such, since we did not have access to samples immediately after the bTBI and had few samples within 1 month after injury. In summary, we may speculate that in the months following a bTBI, brain clearance is slowed down to the extent that serum biomarker levels are primarily regulated by a reduced glympahtic flow kinetic, previously reported in Alzheimer disease in animal models and following sleep deprivation, whereas in hours or days after a TBI, direct impact to the nervous tissue might underlie the primary source of serum biomarkers. Given that human studies of brain tissue are highly invasive, identifying a lowinvasive biomarker for TBI would be of great clinical value. Although it is unclear whether inflammatory cytokine levels are influenced by conventional TBI treatments used in the military, the results of our study should be interpreted with caution, since we did not have data on treatment modalities for the cases in our study population. Additional research in other study populations focusing on bTBI and the chronic phase and including post-diagnostic treatment data will be required to further elucidate the association between serum cytokine levels with longer term follow-up after a TBI diagnosis. If confirmed, these results may suggest that in the long run TBI may slow down either the production of cytokines or the glymphatic clearance of those cytokines, thereby reducing their levels in serum. Detailed characterization of the cytokine milieu in peripheral serum during recovery post-bTBI would elucidate the systemic inflammatory process, may help to monitor the recovery or rehabilitative period, and may possibly identify and evaluate therapeutic interventions.

\section{Supplementary information}

Supplementary information accompanies this paper at https://doi.org/10. 1186/s12974-019-1624-z.

Additional file 1: Table S1. Mean differences between cases controls, pre deployment and post deployment. Table S2. Stratified analyses of ANOVA with single model comparing IL-8 means of pre-post cytokine difference for cases versus controls

\section{Abbreviations}

AFHSB: Armed Forces Health Surveillance Branch; ANOVA: Analysis of variance; BBB: Blood brain barrier; BOP: Blast overpressure; bTBI: Blast traumatic brain injury; CV: Coefficient of variation; DoD: Department of Defense; DVBIC: Defense Veterans Brain Injury Center; GOS: Glasgow Outcome Score; ICD-9: International Classification of Diseases, 9th Edition; IL: Interleukin; MCP: Monocyte chemoattractant protein; MMP: Matrix metallopeptidase; NGF: Nerve growth factor; PTSD: Post-traumatic stress disorder; SAS: Statistical Analysis Software, Inc.; sd: Standard deviation; se: Standard error; TBI: Traumatic brain injury;

TGF: Transferring growth factor; VA: Veterans Administration

\section{Acknowledgements}

The authors would like to acknowledge the contributions to this study by our late colleague, Dr. Vasantha Srikantan. The authors would like to thank the Armed

Forces Health Surveillance Branch for providing serum and relevant data.

\section{Disclaimer}

The content of this publication is the sole responsibility of the authors and does not necessarily reflect the views or policies of the Uniformed Services University of the Health Sciences (USUHS), the Walter Reed Army Institute of Research, Department of Defense (DoD), or the Departments of the Army, Navy or Air Force. Mention of trade names, commercial products, or organizations does not imply endorsement by the US Government.

\section{Authors' contributions}

JR conceived and designed the study. JR and LF acquired the data. JR, LW, and LC created the detailed analysis plan and/or analyzed the data. JR, LL, LW, CB, JK, ZG, and $L F$ interpreted the findings. $L F$ contributed the resources. JR, $L L$, and $L W$ composed the first draft of manuscript. JR, LL, LW, CB, JK, LC, ZG, and LF provided critical revisions and edits (scientific content) to the provisional drafts. JR, LL, LW,

$\mathrm{CB}, \mathrm{JK}, \mathrm{LC}, \mathrm{ZG}$, and $\mathrm{LF}$ reviewed and approved the final version for submission.

\section{Funding}

This study was funded by a Department of Defense (DOD) Psychological Health/Traumatic Brain Injury ( $\mathrm{PH} / \mathrm{TBI})$ Research Programs of the Office of the Congressionally Directed Medical Research Programs (CDMRP) Grant: PT073882. This grant funded the collection and analysis of the data. This research was partially supported (ZG) by a grant from U.S. Department of Defense, Program Project 308430 USUHS and Collaborative Health Initiative Research Program (NHLBI/NIH and USUHS).

\section{Availability of data and materials}

The datasets generated during and/or analyzed during the current study are not publicly available due to their being based on US military data but are available from the corresponding author on reasonable request.

Ethics approval and consent to participate

This study was reviewed by the USU IRB and deemed non-human subject research.

Consent for publication

Not applicable

\section{Competing interests}

The authors declare that they have no competing interests.

\section{Author details}

${ }^{1}$ Department of Preventive Medicine and Biostatistics, F. Edward Hébert School of Medicine, Uniformed Services University, Bethesda, MD, USA.

${ }^{2}$ Statistics and Epidemiology Branch, Walter Reed Army Institute of Research, Silver Spring, MD, USA. ${ }^{3}$ Department of Pediatrics, F. Edward Hébert School 
of Medicine, Uniformed Services University, Bethesda, MD, USA. ${ }^{4}$ Department of Anatomy, Physiology, and Genetics, F. Edward Hébert School of Medicine, Uniformed Services University, Bethesda, MD, USA. ${ }^{5}$ National Intrepid Center of Excellence, Walter Reed National Military Medical Center, Bethesda, MD, USA.

Received: 7 May 2019 Accepted: 28 October 2019

Published online: 13 January 2020

\section{References}

1. AFHSC. DoD TBI Statistics 2000-2013. Washington, DC: DoD; 2013.

2. Masel BE, Bell RS, Brossart S, Grill RJ, Hayes RL, Levin HS, et al. Galveston brain injury conference 2010: clinical and experimental aspects of blast injury. J Neurotrauma. 2012;29(12):2143-71. https://doi.org/10.1089/neu. 2011.2258.

3. Cernak I, Noble-Haeusslein L. Traumatic brain injury: an overview of pathobiology with emphasis on military populations. J Cereb Blood Flow Metab. 2010;30(2):255-66. https://doi.org/10.1038/jcbfm.2009.203.

4. DePalma RG, Burris DG, Champion HR, Hodgson MJ. Blast injuries. N Engl J Med. 2005;352(13):1335-42. https://doi.org/10.1056/NEJMra042083.

5. Kobeissy F, Mondello S, Tumer N, Toklu HZ, Whidden MA, Kirichenko N, et al. Assessing neuro-systemic \& behavioral components in the pathophysiology of blast-related brain injury. Front Neurol. 2013;4:186. https://doi.org/10.3389/fneur.2013.00186.

6. Mayorga MA. The pathology of primary blast overpressure injury. Toxicology. 1997;121(1):17-28.

7. Moore DF, Jaffee MS. Military traumatic brain injury and blast. NeuroRehabilitation. 2010;26(3):179-81. https://doi.org/10.3233/NRE-20100553.

8. Warden DL, French LM, Shupenko L, Fargus J, Riedy G, Erickson ME, et al. Case report of a soldier with primary blast brain injury. Neuroimage. 2009; 47(Suppl 2):T152-3. https://doi.org/10.1016/j.neuroimage.2009.01.060 Epub 2009 Feb 1010.

9. Chen $Y$, Huang W, Constantini S. The differences between blast-induced and sports-related brain injuries. Front Neurol. 2013;4:119. https://doi.org/10. 3389/fneur.2013.00119.

10. French LM. Military traumatic brain injury: an examination of important differences. Ann N Y Acad Sci. 2010;1208:38-45. https://doi.org/10.1111/j. 1749-6632.2010.05696.x

11. Carr W, Polejaeva E, Grome A, Crandall B, LaValle C, Eonta SE, Young LA. Relation of repeated low-level blast exposure with symptomology similar to concussion. J Head Trauma Rehabil. 2015;30(1):47-55. https://doi.org/10. 1097/HTR.0000000000000064.

12. Elder GA, Stone JR, Ahlers ST. Effects of low-level blast exposure on the nervous system: is there really a controversy? Front Neurol. 2014;5:269. https://doi.org/10.3389/fneur.2014.00269 eCollection 02014

13. Nathan DE, Bellgowan JF, Oakes TR, French LM, Nadar SR, Sham EB, et al. Assessing quantitative changes in intrinsic thalamic networks in blast and nonblast mild traumatic brain injury: implications for mechanisms of injury. Brain Connect. 2016:6(5):389-402. https://doi.org/10.1089/brain.2015.0403 Epub 2016 Apr 1020

14. Shively SB, Horkayne-Szakaly I, Jones RV, Kelly JP, Armstrong RC, Perl DP. Characterisation of interface astroglial scarring in the human brain after blast exposure: a post-mortem case series. Lancet Neurol. 2016;15(9):944-53. https://doi.org/10.1016/S1474-4422(16)30057-6 Epub 32016 Jun 30059.

15. Hinson HE, Rowell S, Schreiber M. Clinical evidence of inflammation driving secondary brain injury: a systematic review. J Trauma Acute Care Surg. 2015; 78(1):184.

16. Morganti-Kossmann MC, Satgunaseelan L, Bye N, Kossmann T. Modulation of immune response by head injury. Injury. 2007;38(12):1392-400. https:// doi.org/10.1016/j.injury.2007.10.005.

17. George N, Geller HM. Extracellular matrix and traumatic brain injury. J Neurosci Res. 2018;96(4):573-88. https://doi.org/10.1002/jnr.24151.

18. Woodcock T, Morganti-Kossmann MC. The role of markers of inflammation in traumatic brain injury. Front Neurol. 2013;4(18):4.

19. Anthony DC, Couch Y, Losey P, Evans MC. The systemic response to brain injury and disease. Brain Behav Immun. 2012;26(4):534-40. https:/doi.org/ 10.1016/j.bbi.2011.10.011.

20. Morganti-Kossmann MC, Semple BD, Hellewell SC, Bye N, Ziebell JM. The complexity of neuroinflammation consequent to traumatic brain injury: from research evidence to potential treatments. Acta Neuropathol. 2018; 137:731-55. https://doi.org/10.1007/s00401-018-1944-6.

21. Ziebell JM, Morganti-Kossmann MC. Involvement of pro- and antiinflammatory cytokines and chemokines in the pathophysiology of traumatic brain injury. Neurotherapeutics. 2010;7(1):22-30.

22. Plesnila N. The immune system in traumatic brain injury. Curr Opin Pharmacol. 2016;26:110-7.

23. Wang KKW, Moghieb A, Yang ZH, Zhang ZQ. Systems biomarkers as acute diagnostics and chronic monitoring tools for traumatic brain injury Sensing Technologies for Global Health, Military Medicine, and Environmental Monitoring III (Vol. 8723). Baltimore: Proc SPIE; 2013.

24. Morganti-Kossmann MC, Rancan M, Stahel PF, Kossmann T. Inflammatory response in acute traumatic brain injury: a double-edged sword. Curr Opin Crit Care. 2002;8(2):101-5.

25. Carpenter K, Czosnyka M, Jalloh I, Newcombe V, Helmy A, Shannon R, et al. Systemic, local, and imaging biomarkers of brain injury: more needed, and better use of those already established? Front Neurol. 2015;6:26.

26. Gill J, Motamedi V, Osier N, Dell K, Arcurio L, Carr W, et al. Moderate blast exposure results in increased IL-6 and TNFalpha in peripheral blood. Brain Behav Immun. 2017;65:90-4. https://doi.org/10.1016/j.bbi.2017.02.015.

27. Di Battista AP, Rhind SG, Hutchison MG, Hassan S, Shiu MY, Inaba K, et al. Inflammatory cytokine and chemokine profiles are associated with patient outcome and the hyperadrenergic state following acute brain injury. J Neuroinflammation. 2016;13:40. https://doi.org/10.1186/s12974-016-0500-3.

28. Hayakata T, Shiozaki T, Tasaki O, Ikegawa H, Inoue $Y$, Toshiyuki F, et al. Changes in CSF S100B and cytokine concentrations in early-phase severe traumatic brain injury. Shock. 2004;22(2):102-7.

29. Lustenberger T, Kern M, Relja B, Wutzler S, Störmann P, Marzi I. The effect of brain injury on the inflammatory response following severe trauma. Immunobiology. 2016;221(3):427-31. https://doi.org/10.1016/j.imbio.2015.11. 011.

30. Stein DM, Lindell A, Murdock KR, Kufera JA, Menaker J, Keledjian K, et al. Relationship of serum and cerebrospinal fluid biomarkers with intracranial hypertension and cerebral hypoperfusion after severe traumatic brain injury. J Trauma. 2011;70(5):1096-103. https://doi.org/10.1097/TA. ob013e318216930d

31. Csuka E, Morganti-Kossmann MC, Lenzlinger PM, Joller H, Trentz O, Kossmann T. IL-10 levels in cerebrospinal fluid and serum of patients with severe traumatic brain injury: relationship to IL-6, TNF-alpha, TGF-beta1 and blood-brain barrier function. J Neuroimmunol. 1999;101(2):211-21.

32. Kossmann T, Hans VH, Imhof HG, Stocker R, Grob P, Trentz O, MorgantiKossmann C. Intrathecal and serum interleukin- 6 and the acute-phase response in patients with severe traumatic brain injuries. Shock. 1995;4(5): 311-7.

33. Kumar RG, Boles JA, Wagner AK. Chronic inflammation after severe traumatic brain injury: characterization and associations with outcome at 6 and 12 months postinjury. J Head Trauma Rehabil. 2015;30(6):369-81.

34. Agoston DV, Elsayed M. Serum-based protein biomarkers in blast-induced traumatic brain injury spectrum disorder. Front Neurol. 2012;3:107. https:// doi.org/10.3389/fneur.2012.00107.

35. Elder GA, Gama Sosa MA, De Gasperi R, Stone JR, Dickstein DL, Haghighi F, et al. Vascular and inflammatory factors in the pathophysiology of blastinduced brain injury. Front Neurol. 2015;6:48. https://doi.org/10.3389/fneur. 2015.00048

36. Zou YY, Kan EM, Lu J, Ng KC, Tan MH, Yao L, Ling EA. Primary blast injuryinduced lesions in the retina of adult rats. J Neuroinflammation. 2013;10:79. https://doi.org/10.1186/1742-2094-10-79.

37. Dalle Lucca JJ, Chavko M, Dubick MA, Adeeb S, Falabella MJ, Slack JL, et al. Blast-induced moderate neurotrauma (BINT) elicits early complement activation and tumor necrosis factor alpha (TNFa) release in a rat brain. J Neurol Sci. 2012;318(1-2):146-54. https://doi.org/10.1016/j.jns.2012.02.002.

38. Weckbach S, Perl M, Heiland T, Braumuller S, Stahel PF, Flierl MA, et al. A new experimental polytrauma model in rats: molecular characterization of the early inflammatory response. Mediat Inflamm. 2012;2012:890816. https:// doi.org/10.1155/2012/890816.

39. Iliff JJ, Chen MJ, Plog BA, Zeppenfeld DM, Soltero M, Yang L, et al. Impairment of glymphatic pathway function promotes tau pathology after traumatic brain injury. J Neurosci. 2014;34(49):16180-93. https://doi.org/10. 1523/jneurosci.3020-14.2014.

40. Plog BA, Dashnaw ML, Hitomi E, Peng W, Liao Y, Lou N, et al. Biomarkers of traumatic injury are transported from brain to blood via the glymphatic 
system. J Neurosci. 2015;35(2):518-26. https://doi.org/10.1523/jneurosci. 3742-14.2015.

41. Absinta M, Ha SK, Nair G, Sati P, Luciano NJ, Palisoc M, et al. Human and nonhuman primate meninges harbor lymphatic vessels that can be visualized noninvasively by MRI. Elife. 2017;6:e29738. https://doi.org/10.7554/ eLife.29738.

42. Ha SK, Nair G, Absinta M, Luciano NJ, Reich DS. Magnetic resonance imaging and Histopathological visualization of human Dural lymphatic vessels. Bio Protoc. 2018;8(8):e2819. https://doi.org/10.21769/BioProtoc.2819.

43. Rasmussen MK, Mestre $\mathrm{H}$, Nedergaard M. The glymphatic pathway in neurological disorders. Lancet Neurol. 2018;17(11):1016-24. https://doi.org/ 10.1016/s1474-4422(18)30318-1.

44. Surbatovic M, Filipovic N, Radakovic S, Stankovic N, Slavkovic Z. Immune cytokine response in combat casualties: blast or explosive trauma with or without secondary sepsis. Mil Med. 2007:172(2):190-5.

45. Devoto C, Arcurio L, Fetta J, Ley M, Rodney T, Kanefsky R, Gill J. Inflammation relates to chronic behavioral and neurological symptoms in military with traumatic brain injuries. Cell Transplant. 2016;26:1169-77. https://doi.org/10.3727/096368916×693455.

46. Dash PK, Zhao J, Hergenroeder G, Moore AN. Biomarkers for the diagnosis, prognosis, and evaluation of treatment efficacy for traumatic brain injury. Neurotherapeutics. 2010;7(1):100-14. https://doi.org/10.1016/j.nurt.2009.10. 019.

47. Hellewell S, Semple BD, Morganti-Kossmann MC. Therapies negating neuroinflammation after brain trauma. Brain Res. 2016;1640(Part A):36-56. https://doi.org/10.1016/j.brainres.2015.12.024.

48. Kulbe JR, Geddes JW. Current status of fluid biomarkers in mild traumatic brain injury. Exp Neurol. 2016;275(Part 3):334-52. https://doi.org/10.1016/j. expneurol.2015.05.004.

49. Rubertone MV, Brundage JF. The defense medical surveillance system and the Department of Defense Serum Repository: glimpses of the future of public health surveillance. Am J Public Health. 2002;92(12):1900-4.

50. VA/DoD. Clinical practice guideline for Management of Concussion/mild traumatic brain injury. J Rehabil Res Dev. 2009:46(6):CP1-68.

51. Institute, S. TheSAS system for windows, part 9.3. Cary: SAS Institute; 2012.

52. Rodney T, Osier N, Gill J. Pro- and anti-inflammatory biomarkers and traumatic brain injury outcomes: a review. Cytokine. 2018;110:248-56. https://doi.org/10.1016/j.cyto.2018.01.012.

53. Ondruschka B, Schuch S, Pohlers D, Franke H, Dressler J. Acute phase response after fatal traumatic brain injury. Int J Legal Med. 2018;132(2):5319. https://doi.org/10.1007/s00414-017-1768-2.

54. Abdul-Muneer PM, Pfister BJ, Haorah J, Chandra N. Role of matrix Metalloproteinases in the pathogenesis of traumatic brain injury. Mol Neurobiol. 2016;53(9):6106-23. https://doi.org/10.1007/s12035-015-9520-8.

55. Santarsieri M, Kumar RG, Kochanek PM, Berga S, Wagner AK. Variable neuroendocrine-immune dysfunction in individuals with unfavorable outcome after severe traumatic brain injury. Brain Behav Immun. 2015;45: 15-27. https://doi.org/10.1016/j.bbi.2014.09.003.

56. Hoesel B, Schmid JA. The complexity of NF-kappaB signaling in inflammation and cancer. Mol Cancer. 2013;12:86. https://doi.org/10.1186/ 1476-4598-1112-1186.

57. Yamaguchi K, Kumakura S, Murakami T, Someya A, Inada E, Nagaoka I. Ketamine suppresses the substance P-induced production of IL-6 and IL-8 by human U373MG glioblastoma/astrocytoma cells. Int J Mol Med. 2017; 39(3):687-92. https://doi.org/10.3892/ijmm.2017.2875 Epub 2017 Feb 3893.

58. Bian XX, Yuan XS, Qi CP. Effect of recombinant human erythropoietin on serum S100B protein and interleukin-6 levels after traumatic brain injury in the rat. Neurol Med Chir (Tokyo). 2010;50(5):361-6.

59. Bingham D, John CM, Levin J, Panter SS, Jarvis GA. Post-injury conditioning with lipopolysaccharide or lipooligosaccharide reduces inflammation in the brain. J Neuroimmunol. 2013;256(1-2):28-37. https://doi.org/10.1016/j. jneuroim.2012.12.009 Epub 2013 Jan 1017.

60. Calvalido J, Wood GA, Mutsaers AJ, Wood D, Sears W, Woods JP. Comparison of serum cytokine levels between dogs with multicentric lymphoma and healthy dogs. Vet Immunol Immunopathol. 2016;182:10614. https://doi.org/10.1016/i.vetimm.2016.10.009 Epub 2016 Oct 1019.

61. Keustermans GC, Hoeks SB, Meerding JM, Prakken BJ, de Jager W. Cytokine assays: an assessment of the preparation and treatment of blood and tissue samples. Methods. 2013;61 (1):10-7. https://doi.org/10.1016/j.ymeth.2013.04. 005 Epub 2013 Apr 1019.
62. Wang J, Zhu HH, Xue JH, Wu SS, Chen Z. Effects of storage conditions on the stability of serum CD163, NGAL, HMGB1 and MIP2. Int J Clin Exp Pathol. 2015;8(4):4099-105 eCollection 2015.

63. Graham C, Chooniedass R, Stefura WP, Lotoski L, Lopez P, Befus AD, et al. Stability of pro- and anti-inflammatory immune biomarkers for human cohort studies. J Transl Med. 2017;15(1):53. https://doi.org/10.1186/s1296712017-11154-12963.

64. Talsky A, Pacione $L R$, Shaw $T$, Wasserman $L$, Lenny A, Verma A, et al. Pharmacological interventions for traumatic brain injury. BCMJ. 2011;53(1): 26-31.

65. Baumann CR, Werth E, Stocker R, Ludwig S, Bassetti CL. Sleep-wake disturbances 6 months after traumatic brain injury: a prospective study. Brain. 2007;130(Pt 7):1873-83. https://doi.org/10.1093/brain/awm109.

66. Sullan MJ, Asken BM, Jaffee MS, Dekosky ST, Bauer RM. Glymphatic system disruption as a mediator of brain trauma and chronic traumatic encephalopathy. Neurosci Biobehav Rev. 2018;84:316-24. https://doi.org/10. 1016/j.neubiorev.2017.08.016.

\section{Publisher's Note}

Springer Nature remains neutral with regard to jurisdictional claims in published maps and institutional affiliations.

\section{Ready to submit your research? Choose BMC and benefit from:}

- fast, convenient online submission

- thorough peer review by experienced researchers in your field

- rapid publication on acceptance

- support for research data, including large and complex data types

- gold Open Access which fosters wider collaboration and increased citations

- maximum visibility for your research: over $100 \mathrm{M}$ website views per year

At BMC, research is always in progress.

Learn more biomedcentral.com/submissions 